\title{
Long-Term Follow-Up and Oncological Outcome of Patients Undergoing Radical Cystectomy for Bladder Cancer following an Enhanced Recovery after Surgery (ERAS) Protocol: Results of a Large Randomized, Prospective, Single-Center Study
}

\author{
Brigitte Katharina Ziegelmueller ${ }^{\mathrm{a}} \quad$ Jan-Friedrich Jokisch ${ }^{\mathrm{a}} \quad$ Alexander Buchner $^{\mathrm{a}}$ Tobias Grimm ${ }^{\mathrm{a}}$ \\ Alexander Kretschmer ${ }^{a}$ Gerald Bastian Schul ${ }^{a}$ Christian Stief $^{a} \quad$ Alexander Karl $^{b}$

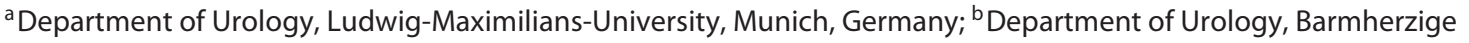 \\ Brüder Hospital, Munich, Germany
}

\section{Keywords}

Cystectomy · Bladder cancer - Early recovery after surgery .

Quality of life · Long-term follow-up

\begin{abstract}
Introduction: Enhanced recovery after surgery (ERAS) concepts are implemented in various surgical disciplines. For patients undergoing radical cystectomy, prospective data are still rare. In the year 2014, our group could prove significant benefits of ERAS for these patients in a prospective randomized study compared to a conservative regimen. Objective: To evaluate long-term follow-up results of ERAS concepts in patients undergoing radical cystectomy for bladder cancer. Methods: Of the 101 patients who had initially prospectively been randomized to ERAS or a conservative regimen, $35 \mathrm{pa}-$ tients could be included in the study. Median follow-up time was 83 months. Primary end point assessed quality of life. Secondary end points were cancer-specific survival and overall survival. Results: Five to seven years after the initial inclusion to the study, no significant difference regarding quality of life parameters was detected ( $p$ values range between 0.112 and 0.970 ). Continence status in patients with neobladder showed no significant differences between
\end{abstract}

ERAS and conservative regimens $(p=0.785)$. Cancer-specific survival in the ERAS group did not differ significantly from that in the conservative group ( 49 vs. $58 \%, p=0.725$ ). Conclusions: While ERAS represents an excellent way to improve postoperative reconvalescence and quality of life in the short-term follow-up, our data do not support the idea that there is also a long-term effect in terms of quality of life issues.

(c) 2019 S. Karger AG, Basel

\section{Introduction}

Enhanced recovery after surgery (ERAS) regimens prevail in several surgical disciplines. ERAS or fasttrack concepts aim to reduce perioperative burden and postoperative morbidity, optimize postoperative reconvalescence, decrease the demand for analgetics, and shorten hospital stay. These approaches were initially presented in colorectal surgery and can be traced back

Brigitte Katharina Ziegelmueller and Jan-Friedrich Jokisch contributed equally to this article.



Dr. med. Brigitte Katharina Ziegelmueller

Department of Urology, Ludwig-Maximilians-University, LMU

Marchioninistrasse 15

DE-81377 Munich (Germany)

E-Mail Brigitte.ziegelmueller@med.uni-muenchen.de 
to the early 1990s. The first study that proved significant benefit in postoperative reconvalescence using fasttrack programs was published in 2002 by Kehlet and Wilmore [1]. A number of subsequent studies showed improved outcome in terms of morbidity, incidence of ileus, reconvalescence, and hospitalization time for patients after colorectal surgery obtaining postoperative care according to such fast-track protocols [2-4]. While ERAS regimens represent the standard of care in colorectal surgery, data are still limited for patients undergoing radical cystectomy for bladder cancer [5-12]. The available studies yielded positive results and demand for further research in this field, as radical cystectomy is the urological procedure related to the highest rate of morbidity, mortality, and hospital stay, and effective concepts for improved outcome should be realized.

For better evaluation of fast-track programs in radical cystectomy, our group performed a prospective randomized study published in 2014. A total of 101 patients who underwent radical cystectomy for bladder cancer between 2010 and 2012 were enrolled. Patients were prospectively randomized into 2 groups, with 39 patients randomized to the conservative group and 62 to the ERAS group. In this study, significant benefits could be demonstrated for patients who underwent radical cystectomy for bladder cancer in the ERAS arm compared to conservative regimen in terms of postoperative morbidity, quality of life, use of analgetics, and time spent at the intermediate care unit [13]. Being extracted in the postoperative setting, data represent short-term effects of ERAS concepts after radical cystectomy.

Quality of life was significantly better in patients of the ERAS group in the short term. So, we wondered if this effect could persist in the long-term period. Better quality of life also could be associated with a higher degree of motivation for pelvic floor muscle training and so lead to better results regarding continence status. As patients after radical cystectomy are followed-up anyway for a long time, it seemed to be interesting to reevaluate the positive effect of ERAS that could be shown in the postoperative setting also in the long-term follow-up. We did not expect an influence of ERAS on oncological outcome such as overall survival or cancerspecific survival.

This study was conducted to reevaluate the included patients in terms of quality of life and oncological outcome in dependence from the initial postoperative management (ERAS vs. conservative regimen) and type of urinary diversion (neobladder vs. ileal conduit).

\section{Materials and Methods}

\section{Patients}

Initially, 101 patients who underwent radical cystectomy for bladder cancer between 2010 and 2012 were enrolled in the study. They had been prospectively randomized into two groups, a conservative and an ERAS group. Three of the 101 patients were lost to follow-up. Of the remaining 98 patients, 52 were still alive and 46 have died. Among the 46 patients who have died, 29 had received the ERAS and 17 the conservative regimen. Among the 52 patients still living, 31 were in the ERAS group and 21 in the conservative group. Of the 52 patients, 35 delivered data of a further follow-up and so could be included in the study. An ileum neobladder was applied in 25 patients and an ileal conduit in 10 patients. Out of the 25 patients with an ileum neobladder, 16 were in the ERAS group and 9 in the conservative group.

Out of the 35 patients, 21 (60\%) received postoperative management according to the ERAS protocol and 14 (40\%) conservative management. Terminal ileum had been used for urinary diversion. Method of surgery was open radical cystectomy. $14 \%$ of them were female $(5 \%, n=5)$ and $86 \%$ were male $(95 \%, n=30)$. Median follow-up of all the 98 patients was 39.5 months (IQR 15-60). Median follow-up of the 35 patients whose questionnaires could be used for further evaluation was 83 months (IQR 78-89). Median age of the patients in the ERAS group was 70 years (IQR 62.5-75) and in the conventional group 67.5 years (IQR 57-77). Difference was not significant $(p=0.439)$.

The primary end point was the assessment of quality of life parameters. The validated questionnaire EORTC QLQ-C30 was used. Mann-Whitney $U$ test was applied for evaluation of the QLQ-30 questionnaire.

Secondary end point was the evaluation of continence in the neobladder group by using ICIQ-UI and comparison between ERAS and conventional perioperative program. Another secondary end point was the evaluation of overall and cancer-specific survival in the patients respecting also the patients who were already deceased during the follow-up period. A possible correlation to perioperative regimen (ERAS vs. conservative) was evaluated by applying log-rank test. Cancer-specific survival was evaluated in all patients (46 deceased and 52 living patients) and was compared between the ERAS and the conservative group.

\section{Description of Tested Regimens}

In the ERAS protocol, no extended retrograde or antegrade bowel preparation was used. In the afternoon before the operation, 2 units of bisacodyl were administered orally to stimulate bowel movement. High-calorie protein-based drinks were offered at least three times per day before surgery. Two hours before the operation, clear fluids and high-calorie drinks were offered.

The stomach tube was removed right after surgery. Bowel movement was stimulated at the first postoperative day using magnesium orally ( $200 \mathrm{mg}$ per unit) 3 times a day. Mobilization was conducted as early as possible on the day of surgery. Removal of drains was performed at the first or second postoperative day. Analgetic drugs were offered in both groups equally.

The conservative management included an orthograde bowel preparation on the day before surgery. The stomach tube remained in place for 2 or 3 days after surgery until first bowel movement was registered. Enteralization with solid food was started after a sufficient bowel movement was documented. Fluids were given on 
the first postoperative day in restricted amounts. If bowel movement was retarded, stimulation was performed by using neostigmine, metoclopramide, and dexpanthenol on the second postoperative day.

\section{Results}

\section{Primary End Point: Quality of Life}

There was no significant difference regarding life quality assessed by EORTC questionnaire QLQ-30 ( $p=0.211-$ $0.970)$. Median global health score was 83 in the conventional group and 79 in the ERAS group $(p=0.211)$. Physical functioning score was 83 in both groups $(p=0.773)$. Role functioning score was 83 in the conventional group and 75 in the ERAS group $(p=0.782)$. Emotional functioning score was 96 in the conventional group and 75 in the ERAS group $(p=0.162)$. Cognitive functioning score showed a value of 100 for the conventional group and 92 for the ERAS group $(p=0.778)$. Social functioning score was 83 in both groups $(p=0.539)$. Fatigue score was 89 in the conventional and 67 in the ERAS group $(p=0.684)$. Nausea and vomiting score was 100 in both groups $(p=0.305)$, and pain score $(p=$ $0.118)$ and dyspnea score $(p=0.970)$ showed the same results. Insomnia score showed values of 67 (conservative group) and 83 (ERAS group) ( $p=0.213$ ). Appetite loss, constipation, diarrhea, and financial difficulties scores were 100 in each group $(p=0.112,0.460,0.364,0.906)$. Table 1 offers an overview of the functional scales and symptom scales in each group. None of the differences were significant.

\section{Secondary End Points}

Continence was compared within the neobladder group by the ICIQ questionnaire. No significant differences could be observed between the ERAS and conservative regimen. Median ICIQ score was 7 in the ERAS and 5.5 in the conservative group ( $p=0.785)$.

Cancer-specific survival in the ERAS group was compared to that in the conservative group. $45.5 \%$ of the patients initially enrolled in the study were already deceased (46 out of 98 patients). Twenty-nine had received the ERAS and 17 patients the conservative regimen. Cause of death was cancer in $73.9 \%$ of patients (34 out of the 46 patients), cardiac causes in 2 patients $(4.3 \%)$, other reasons were found in 3 patients $(6.5 \%)$, and cause of death was unknown in 7 patients (15.2\%). Thus, among patients who had died during the follow-up period after radical cystectomy for bladder cancer, tumor was responsible for death in most of the cases. This finding is in accordance with higher tumor stages present in the specimens of the patients who were deceased. The patients who had died
Table 1. Overview of the parameters of the QLQ-30 questionnaire according to EORTC manual

\begin{tabular}{lccc}
\hline Parameter & $\begin{array}{l}\text { Median } \\
\text { ERAS }\end{array}$ & $\begin{array}{l}\text { Median } \\
\text { conventional }\end{array}$ & $p$ value \\
\hline Global health score & 79 & 83 & 0.211 \\
PF & 83 & 83 & 0.773 \\
RF & 75 & 83 & 0.782 \\
EF & 75 & 96 & 0.162 \\
CF & 92 & 100 & 0.778 \\
SF & 83 & 83 & 0.539 \\
FA & 67 & 89 & 0.684 \\
NV & 100 & 100 & 0.305 \\
PA & 100 & 100 & 0.118 \\
DY & 100 & 100 & 0.970 \\
SL & 83 & 67 & 0.213 \\
AP & 100 & 100 & 0.112 \\
CO & 100 & 100 & 0.460 \\
DI & 100 & 100 & 0.364 \\
FI & 100 & 100 & 0.906 \\
\hline
\end{tabular}

The score of each parameter ranges from 0 to 100 , while 100 is the best score. Comparison of the groups was calculated by MannWhitney U test.

had higher tumor stages as well as positive lymph nodes, metastases, and local recurrences more frequently than the patients who are still alive.

T3 stage could be found in 29 specimens among the patients who had died (21 pT3) and only in 12 patients who are still alive (12 pT3). Stage T4a could even be found in 9 patients who were already deceased and only in 2 patients who are still alive. The patients who were deceased had positive lymph nodes to a larger proportion (19 patients vs. 6 patients who were still living). Development of metastases during the follow-up period could be observed in 6 patients who had died (1 patient with lung metastases, 3 patients with bone metastases, and 2 patients with liver metastases) and in 4 patients who were still living ( 3 patients with lung and 1 patient with bone metastases). Pn1 and L1 status had been found more frequently in patients who had already died (17 Pn1 and 16 L1 vs. 4 Pn1 and 7 L1). Surgical margin was positive more frequently in patients who had died than in patients who were still alive (9 R1 and 1 R2 vs. 3 R1 and 0 R2). Regarding further therapies, 1 patient had received neoadjuvant chemotherapy and 1 patient adjuvant radiotherapy among the group of patients who had died. Three patients developed local recurrence, none among the patients still living. Table 2 gives an overview of the pathologic features of the two groups. 
Table 2. Overview of pathologic features as well as presence of metastases, recurrence, and further therapies in the patients who had died during the follow-up period compared to the patients who were still living: advanced tumor stages and positive lymph node status, as well as distant metastases and local recurrence were observed more frequently in the deceased patients

\begin{tabular}{|c|c|c|}
\hline & $\begin{array}{l}\text { Patients } \\
\text { alive } \\
(n=52)\end{array}$ & $\begin{array}{l}\text { Deceased } \\
\text { patients } \\
(n=46)\end{array}$ \\
\hline \multicolumn{3}{|l|}{ T stage } \\
\hline $\mathrm{Tx}$ & 2 & 1 \\
\hline T0 & 7 & 0 \\
\hline $\mathrm{Ta}$ & 1 & 1 \\
\hline Tis & 6 & 2 \\
\hline $\mathrm{T} 1$ & 3 & 1 \\
\hline $\mathrm{T} 2$ & 16 & 8 \\
\hline T3 & 12 & 21 \\
\hline $\mathrm{T} 4 \mathrm{a}$ & 2 & 9 \\
\hline \multicolumn{3}{|l|}{ Nodal status } \\
\hline $\mathrm{pN} 1$ & 6 & 19 \\
\hline $\mathrm{pNx}$ & 4 & 5 \\
\hline pN0 & 0 & 0 \\
\hline \multicolumn{3}{|c|}{ Development of metastases during follow-up } \\
\hline $\mathrm{M}+$ & 4 & 6 \\
\hline Lung metastases & 3 & 1 \\
\hline Bone metastases & 1 & 3 \\
\hline Liver metastases & 0 & 2 \\
\hline \multicolumn{3}{|l|}{ Pn, L status } \\
\hline Pn1 & 4 & 17 \\
\hline $\mathrm{L} 1$ & 7 & 16 \\
\hline \multicolumn{3}{|l|}{ Resection status } \\
\hline $\mathrm{R} 1$ & 3 & 9 \\
\hline $\mathrm{R} 2$ & 0 & 1 \\
\hline \multicolumn{3}{|l|}{ Further therapies } \\
\hline Neoadjuvant chemotherapy & 0 & 1 \\
\hline Neoadjuvant radiotherapy & 0 & 0 \\
\hline Adjuvant chemotherapy & 0 & 1 \\
\hline Chemotherapy in the course & 0 & 0 \\
\hline Adjuvant radiotherapy & 0 & 1 \\
\hline \multicolumn{3}{|l|}{ Recurrence } \\
\hline Distant recurrence & 0 & $\begin{array}{l}4 \text { ( } 2 \text { bone, } \\
2 \text { liver metastases) }\end{array}$ \\
\hline Local recurrence & 0 & 3 \\
\hline
\end{tabular}

Considering cancer-specific survival in respect to different perioperative regimens, no significant difference could be observed between the ERAS and the conservative group (see Fig. 1a). Five-year survival rate was $49 \%$ in the ERAS group and $58 \%$ in the conservative group $(p=0.725)$. Figure $1 \mathrm{~b}$ does not show a significant difference in overall survival in respect to different perioperative regimens $(p=0.550)$.

\section{Discussion}

The underlying study was a prospective randomized study which included a large volume of patients who had undergone radical cystectomy for bladder cancer and could prove significant benefits of ERAS in a variety of quality of life parameters over conservative regimen [13]. Hence, this study could prove that ERAS was a viable concept for postoperative care after radical cystectomy in patients with bladder cancer and suggests ERAS to be established as the standard of postoperative care. One limitation of this study was the missing data on long-term effects of ERAS, which has been collected for our present study.

To the best of our knowledge, there are no studies in urology or other surgical disciplines on the long-term outcome after the use of ERAS protocols. Some shortterm data on ERAS clearly demonstrates benefits of ERAS in patients undergoing radical cystectomy for bladder cancer. Several studies on short-term effects of ERAS protocols after radical cystectomy for bladder cancer led to similar results and even focused on some further intra- or perioperative factors.

The most recent prospective randomized study regarding this topic included 27 patients undergoing radical cystectomy for bladder cancer. Twelve patients were randomized to follow ERAS protocol and 15 patients to conservative treatment. The results indicated a significant benefit of the ERAS protocol regarding hospital stay, bowel movement, and pain [14]. Patients following ERAS had a significantly shorter hospital stay and time to bowel movement and lower pain scores in the postoperative setting. The small number of patients is a limiting factor of this study.

Further benefits of the ERAS program were described by Pang et al. [15], who evaluated effectiveness of this regimen in relation to intraoperative and perioperative parameters including a large number of patients (433 patients in total and 393 with ERAS). They reported a lower intraoperative blood loss, lower transfusion rates, and faster discharge of patients after radical cystectomy with ERAS.

Similar results regarding intraoperative blood loss could be demonstrated by Ortega-Lucea et al. [12]. They examined 48 patients following ERAS and 51 with conservative regimen. Less intraoperative bleeding and postoperative complications as well as shorter hospital stay were observed in the ERAS group $(n=48)$ compared to the conservative group $(n=51)$.

Baack Kukreja et al. [9] conducted a non-randomized quasi-experimental study including 79 patients with a 


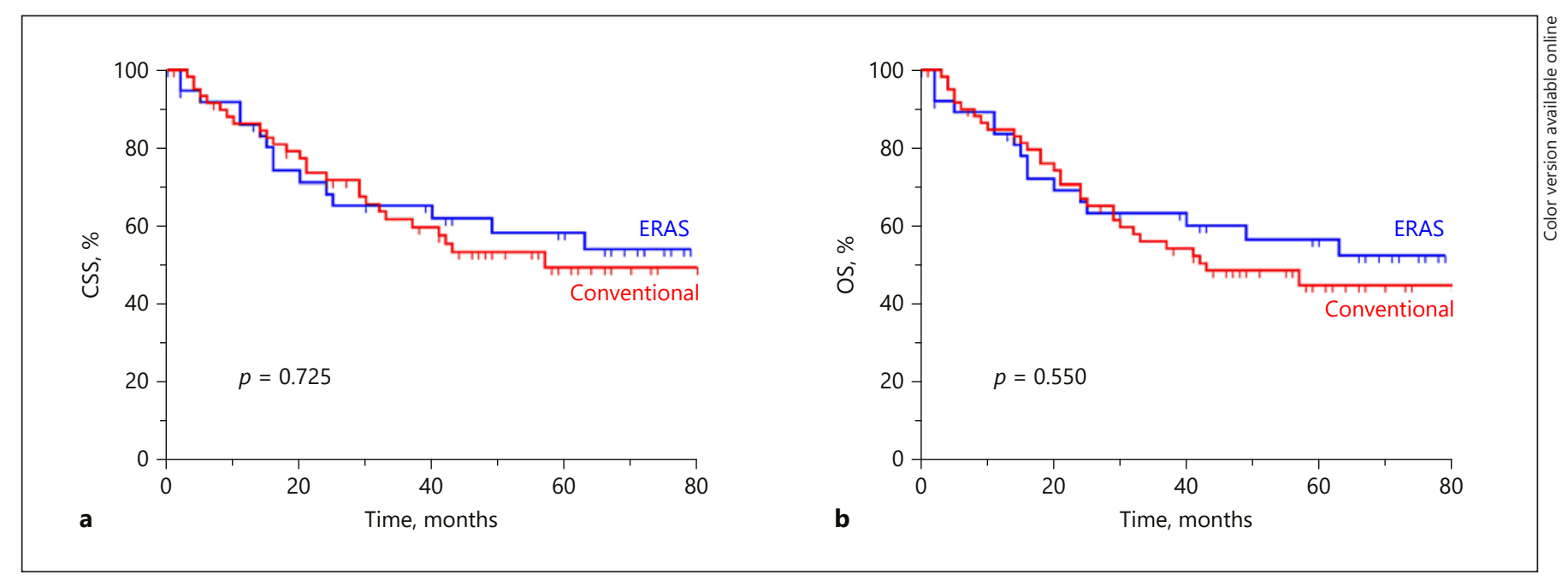

Fig. 1. a Cancer-specific survival (CSS) is better in the ERAS group than in the conventional group after a followup of 30 months. The difference is not statistically significant $(p=0.725)$. b Overall survival (OS) seems to be better in the ERAS group, but the difference is not statistically significant $(p=0.550)$.

concept similar to ERAS (cystectomy enhanced recovery pathway; CERP) and 121 without CERP and revealed a significant shorter duration of hospital stay in the CERP group $(p<0.001)$.

Casans-Francés et al. [10] performed a cohort-comparative study including 41 patients receiving postoperative treatment according to ERAS protocols and 97 historical regimens. There were no differences in complication rate and mortality but a significant lower need for nasogastric tube insertions $(p<0.001)$, parenteral nutrition $(p<0.001)$, and time under endotracheal intubation since anesthesia induction $(p<0.001)$.

Guan et al. [11] examined short-term outcome of laparoscopic radical cystectomy and ileal conduit diversion for bladder cancer patients with ERAS $(n=60)$ in comparison to conservative protocols $(n=55)$ and indicated a significantly shorter duration to first bowel movement, shorter time to regular diet, shorter hospital stay, less complications, and lower white blood cell count and serum of C-reactive protein.

Most of the studies proved earlier discharge from hospital, shorter duration to first bowel movement, less pain, and even less intraoperative complications such as bleeding. Thus, the beneficial short-term effects of ERAS could be confirmed in a number of studies following our underlying prospective study. Indeed, none of them focused on quality of life parameters as we did in our initial study. In the end, quality of life is an essential aspect for the patients' well-being and is possibly influenced by all of these parameters observed peri- and postoperatively. This ob- servation raises the question whether the benefit to quality of life still is obvious in the long term.

To our knowledge, there have been no studies evaluating long-term effects of ERAS in patients after radical cystectomy for bladder cancer up to now. In our study, no significant differences were observed between the ERAS and the conservative group regarding quality of life in the long-term follow-up after a median time of 83 months. The assessment was performed by QLQ-30 questionnaire. A current study which assessed postoperative quality of life of 152 patients with a median follow-up of 48 months revealed preoperative ECOG status, surgeon experience, and daytime incontinence as independent prognostic features for a good postoperative quality of life [16]. The impact of postoperative regimen on quality of life has not been examined so far. It is comprehensible that the postoperative management influences reconvalescence in the short term. After a median follow-up of 83 months, a variety of further aspects might influence life quality, such as comorbidities, physical and psychical health, and social environment, much more than the initial postoperative treatment.

Comparing neobladder and ileal conduit diversion, no difference regarding quality of life was present. Findings over the correlation between form of urinary diversion and quality of life are controversial. A recent meta-analysis that included 10 papers showed a significantly better health-related quality of life in patients with orthotopic neobladder [17]. However, a number of further studies support our results. Cerruto et al. [18] analyzed quality of 
life parameters after orthotopic neobladder versus ileal conduit and showed similar results in terms of global health status between the two forms of urinary diversion. A review that included 29 studies indicated comparable quality of life in either continent or incontinent urinary diversion [19].

Continence was not different between the ERAS and the conservative group for the patients with orthotopic neobladder. No further study has evaluated a possible correlation so far. Daytime continence has been reported to be better in patients with good performance status, without coronary heart disease, and with retained sensation of orthotopic ileal neobladder [20]. The postoperative regimen is not expected to influence continence situation in the long term.

Cancer-specific survival did not differ significantly between the two groups. Pang et al. [15] showed similar results. Histopathological parameters and survival outcomes were not associated with the use of ERAS. Oncological outcome much more depends on oncological parameters, tumor stage, presence of metastases, and resection status. This correlation is proven by our results, which showed higher tumor stages in patients who deceased during the follow-up period.

A limitation of the study is the small number of patients. Due to the long period of time between the enrollment of patients for the initial study and the evaluation of long-term data, a high percentage of patients already deceased. This is the reason why the study on the long-term data can no longer be considered as a large one. It might be difficult to evaluate long-term data of a larger cohort of patients, as most of them are already of an old age when surgery takes place. Nevertheless, larger studies on this topic should be conducted in the future.

This is the first study on long-term effects of ERAS concepts in patients after radical cystectomy for bladder cancer. ERAS had no impact on long-term quality of life, continence, and cancer-specific survival. The main limitation of our study was the small number of patients that could be included, as almost half of them of them were no longer alive at the time of evaluation.

\section{Conclusion}

While ERAS represents an excellent way to improve postoperative reconvalescence and quality of life in the short-term follow-up of patients undergoing radical cystectomy, our data do not support the idea that there is also

a long-term effect in terms of quality of life issues. Also, long-term oncological and functional parameters do not seem to be influenced by the use of perioperative ERAS regimens. Further studies are needed to study the longterm influence of regimens such as ERAS in patients undergoing radical cystectomy or other major surgical procedures.

\section{Acknowledgement}

I would like to thank Prof. Dr. med. Alexander Karl and PD Dr. med. Alexander Buchner.

\section{Statement of Ethics}

This study complies with the guidelines for human studies and is in accordance with the World Medical Association Declaration of Helsinki. Subjects have given their written informed consent. The study protocol was approved by the institute's committee on human research. Ethical approval was not required, as the evaluation was made by questionnaires.

\section{Disclosure Statement}

The authors have no conflicts of interest to declare.

\section{Funding Sources}

There are no funding sources.

\section{Author Contributions}

Brigitte Katharina Ziegelmueller: author. Jan-Friedrich Jokisch: project development, co-first author. Alexander Buchner: data analysis. Tobias Grimm: project development, data management. Alexander Kretschmer: project development. Gerald Schulz: project development. Christian Stief: project development. Alexander Karl: project development, data management, manuscript editing.

References gies to improve surgical outcome. Am J Surg. 2002 Jun;183(6):630-41.

2 Guenaga KF, Matos D, Castro AA, Atallah AN, Wille-Jørgensen P. Mechanical bowel preparation for elective colorectal surgery. Cochrane Database Syst Rev. 2005 Jan;(1): CD001544.

3 Kehlet H. Fast-track colorectal surgery. Lancet. 2008 Mar;371(9615):791-3.
60

Urol Int 2020;104:55-61

DOI: $10.1159 / 000504236$
Ziegelmueller/Jokisch/Buchner/Grimm/ Kretschmer/Schulz/Stief/Karl 
4 Khoo CK, Vickery CJ, Forsyth N, Vinall NS, Eyre-Brook IA. A prospective randomized controlled trial of multimodal perioperative management protocol in patients undergoing elective colorectal resection for cancer. Ann Surg. 2007 Jun;245(6):867-72.

5 Saar M, Ohlmann CH, Siemer S, Lehmann J, Becker F, Stöckle M, et al. Fast-track rehabilitation after robot-assisted laparoscopic cystectomy accelerates postoperative recovery. BJU Int. 2013 Jul;112(2):E99-106.

6 Maffezzini M, Campodonico F, Capponi G, Manuputty E, Gerbi G. Fast-track surgery and technical nuances to reduce complications after radical cystectomy and intestinal urinary diversion with the modified Indiana pouch. Surg Oncol. 2012 Sep;21(3):191-5.

7 Pruthi RS, Nielsen M, Smith A, Nix J, Schultz $\mathrm{H}$, Wallen EM. Fast track program in patients undergoing radical cystectomy: results in 362 consecutive patients. J Am Coll Surg. 2010 Jan;210(1):93-9.

8 Olbert PJ, Baumann L, Hegele A, Schrader AJ, Hofmann R. [Fast-track concepts in the perioperative management of patients undergoing radical cystectomy and urinary diversion: review of the literature and research results]. Urologe A. 2009 Feb;48(2):137-42.

9 Baack Kukreja JE, Kiernan M, Schempp B, Siebert A, Hontar A, Nelson B, et al. Quality Improvement in Cystectomy Care with Enhanced Recovery (QUICCER) study. BJU Int. 2017 Jan;119(1):38-49.
10 Casans-Francés R, Roberto-Alcácer AT, García-Lecina AC, Ferrer-Ferrer ML, Subirá-Ríos J, Guillén-Antón J. Impact of an enhanced recovery after surgery programme in radical cystectomy. A cohort-comparative study. Rev Esp Anestesiol Reanim. 2017 Jun - Jul;64(6): 313-22.

11 Guan X, Liu L, Lei X, Zu X, Li Y, Chen M, et al. A comparative study of fast-track versus [corrected] conventional surgery in patients undergoing laparoscopic radical cystectomy and ileal conduit diversion: chinese experience. Sci Rep. 2014 Oct;4(1):6820.

12 Ortega-Lucea SM, Martínez-Ubieto J, JúdezLegaristi D, Muñoz-Rodriguez L, Gil-Bona J, Pascual-Bellosta AM. The results of implementing a fast-track protocol in radical cystectomy in a tertiary hospital. Actas Urol Esp. 2015 Dec;39(10):620-7.

13 Karl A, Buchner A, Becker A, Staehler M, Seitz M, Khoder W, et al. A new concept for early recovery after surgery for patients undergoing radical cystectomy for bladder cancer: results of a prospective randomized study. J Urol. 2014 Feb;191(2):335-40.

14 Frees SK, Aning J, Black P, Struss W, Bell R, Chavez-Munoz C, et al. A prospective randomized pilot study evaluating an ERAS protocol versus a standard protocol for patients treated with radical cystectomy and urinary diversion for bladder cancer. World J Urol. 2018 Feb;36(2):215-20.

15 Pang KH, Groves R, Venugopal S, Noon AP, Catto JW. Prospective Implementation of Enhanced Recovery After Surgery Protocols to Radical Cystectomy. Eur Urol. 2017 Aug; S0302-2838(17)30660-7.
16 Kretschmer A, Grimm T, Buchner A, Stief CG, Karl A. Prognostic features for quality of life after radical cystectomy and orthotopic neobladder. Int Braz J Urol. 2016 Nov-Dec; 42(6):1109-20.

17 Cerruto MA, D'Elia C, Siracusano S, Porcaro $A B$, Cacciamani G, De Marchi D, et al. Is Health-Related Quality of Life after Radical Cystectomy Using Validated Questionnaires Really Better in Patients with Ileal Orthotopic Neobladder Compared to Ileal Conduit: A Meta-Analysis of Retrospective Comparative Studies. Curr Urol. 2017 Jul;10(2):57-68.

18 Cerruto MA, D’Elia C, Siracusano S, Saleh O, Gacci M, Cacciamani G, et al. Health-related Quality of Life After Radical Cystectomy: A Cross-sectional Study With Matched-pair Analysis on Ileal Conduit vs Ileal Orthotopic Neobladder Diversion. Urology. 2017 Oct; 108:82-9.

19 Yang LS, Shan BL, Shan LL, Chin P, Murray $\mathrm{S}$, Ahmadi N, et al. A systematic review and meta-analysis of quality of life outcomes after radical cystectomy for bladder cancer. Surg Oncol. 2016 Sep;25(3):281-97.

20 Kretschmer A, Grimm T, Buchner A, Grimm J, Grabbert M, Jokisch F, et al. Prognostic Features for Objectively Defined Urinary Continence after Radical Cystectomy and Ileal Orthotopic Neobladder in a Contemporary Cohort. J Urol. 2017 Jan;197(1):210-5. 